\title{
Single-Longitudinal-Mode Fiber Ring Lasers With Taper-Coupled Double-Microsphere-Cavities
}

\author{
Hongdan Wan ${ }^{\circledR}$, Linqian Liu, Zuoqin Ding, Jie Wang, Kaidi Lu, Zuxing Zhang ${ }^{\circledR}$, and Lin Zhang
}

\begin{abstract}
This letter proposes and demonstrates a fiber ring laser using taper-coupled double-microsphere-cavities (DMCs) to achieve single-longitudinal-mode operation. Whispering-gallerymode (WGM) intensity distributions and transmission spectra of the DMC with different microsphere diameters are investigated both theoretically and experimentally. Due to the Vernier effect, the DMC can produce WGM spectra with a higher extinction ratio, a higher side-mode-suppression ratio, a larger free spectral range, and a narrower bandwidth, as compared with a singlemicrosphere cavity. A single-longitudinal-mode fiber ring laser operating near $1.5 \mu \mathrm{m}$ with a bandwidth of $<0.01 \mathrm{~nm}$ and a signal-to-background ratio of about $60 \mathrm{~dB}$ is demonstrated using the taper-coupled DMC as an all-fiber mode selector.
\end{abstract}

Index Terms-Fiber laser, single-longitudinal-mode, tapercoupled double-microsphere-cavities, whispering-gallery-mode, Vernier effect.

\section{INTRODUCTION}

A $S$ APPLIED in optical sensing and communication systems, single-longitudinal-mode (SLM) narrowlinewidth fiber lasers are of great interest to be developed because of their high coherence, flexible wavelengths and compact structures [1]-[3]. Various narrow-bandwidth modeselection methods have been developed and introduced into fiber laser cavities in order to achieve SLM operation, such as fiber gratings [4], [5], saturated absorbers [6], tandem all-Fiber Fabry-Pérot micro-cavities and gaincontrolled active compound cavities, which we previously proposed in [7] and [8]. However, most of these filters have BWs boarder than $1 \mathrm{GHz}$ [4]-[7]. Recently, an ultra-narrowbandwidth mode-selection method based on a high-quality $(\mathrm{Q})$ whispering-gallery-mode (WGM) $\mathrm{CaF}_{2}$ microsphere cavity

Manuscript received August 14, 2017; revised October 16, 2017; accepted October 18, 2017. Date of publication October 24, 2017; date of current version November 7, 2017. This work was supported in part by the National Natural Science Foundation of China under Grant 11704199, in part by the National Science Foundation of Jiangsu Province under Grant BK20150858 and Grant BK20161521, in part by the Distinguished Professor Project of Jiangsu under Grant RK002STP14001, in part by the Talents Projects in Nanjing University of Posts and Telecommunications under Grant NY214002 and Grant NY215002, and in part by the STITP of NUPT under Grant XZD2017040. (Corresponding authors: Hongdan Wan; Zuxing Zhang.)

H. Wan, L. Liu, Z. Ding, J. Wang, and K. Lu are with the Advanced Photonic Technology Laboratory, Nanjing University of Posts and Telecommunications, Nanjing 210023, China (e-mail: hdwan@njupt.edu.cn).

Z. Zhang and L. Zhang are with the Advanced Photonic Technology Laboratory, Nanjing University of Posts and Telecommunications, Nanjing 210023, China, and also with the Aston Institute of Photonic Technologies, Aston University, Birmingham B4 7ET, U.K. (e-mail: zxzhang@njupt.edu.cn; 1.zhang@aston.ac.uk).

Color versions of one or more of the figures in this letter are available online at http://ieeexplore.ieee.org.

Digital Object Identifier 10.1109/LPT.2017.2766183
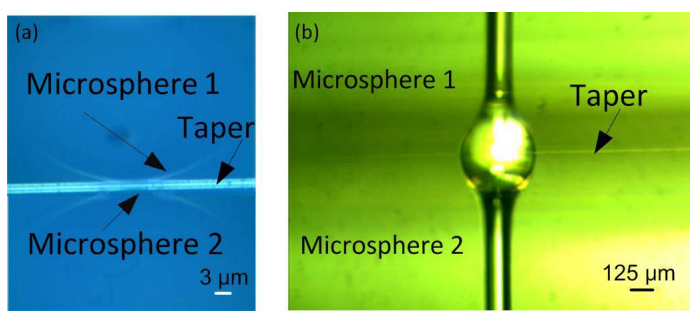

Fig. 1. Microscope images of the taper-coupled DMC: (a) Observed from the horizontal orientation; (b) Observed from the vertical orientation.

was proposed [9]. A SLM laser output with a $650 \mathrm{~Hz}$ linewidth was obtained at $1550 \mathrm{~nm}$. However, manufacturing such a $\mathrm{CaF}_{2}$ microsphere out of a crystal is time- and labor-consuming. Compared to the $\mathrm{CaF}_{2}$ microsphere, a $\mathrm{SiO}_{2}$ microsphere can be easily fabricated by utilizing the surface tension effect [10], [11]. In [11], we proposed and demonstrated a SLM fiber ring laser using a narrow WGM spectrum produced by a single $\mathrm{SiO}_{2}$ microsphere, of which the $\mathrm{Q}$ factor of the microsphere highly depends on the singlemicrosphere-cavity's (SMC) coupling loss and surface quality.

In this letter we propose a SLM selection method based on narrow-bandwidth WGM spectra produced by taper-coupled double-microsphere-cavities (DMC), which consists of two $\mathrm{SiO}_{2}$ microspheres coupling in parallel with a fiber taper. Based on total internal reflection, the WGMs are confined inside the DMC, where the Vernier effect results in WGM spectra with a higher extinction ratio (ER), an increased side-mode-suppression-ratio (SMSR), a compressed BW and a larger free spectral range (FSR), as compared to the SMC. A $1.5 \mu \mathrm{m}$-wavelength SLM fiber ring laser with a $3 \mathrm{~dB}$ bandwidth of $0.01 \mathrm{~nm}$ is experimentally demonstrated using the taper-coupled DMC as the all-fiber mode-selector.

\section{EXPERIMENTAL SETUPS}

Figure 1 shows the microscope image of the taper-coupled DMC with microsphere diameters D1 of about $198.4 \mu \mathrm{m}$ and D2 of about $198.3 \mu \mathrm{m}$, observed by two high-resolution microscope systems in the horizontal and vertical directions. The microsphere diameters are measured at the equatorial plane by the microscope system consisting of a 500-megapixel charge-coupled-device (CCD) camera. The highest resolution and the largest total magnification of the measurement are about $0.6 \mu \mathrm{m}$ and 1600 , respectively. The two microspheres are fabricated by melting the end of a single-mode optical fiber using a $\mathrm{CO}_{2}$ laser. They are placed on two opposite 


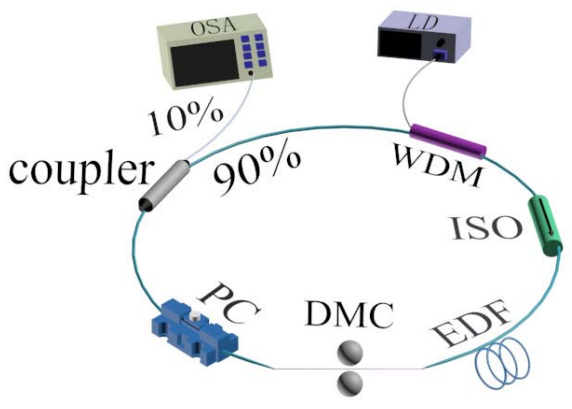

Fig. 2. Experimental setup of the proposed SLM fiber laser stabilized by the taper-coupled DMC.

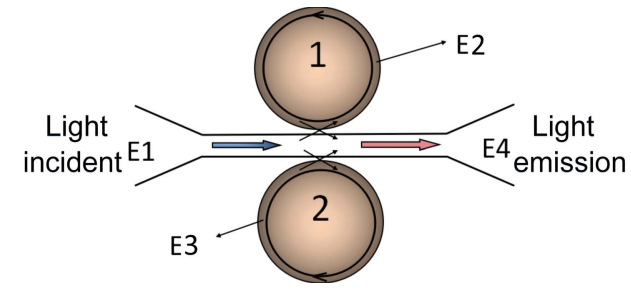

Fig. 3. Light propagation schematic in the taper-coupled DMC.

sides of the tapered fiber. The gap spacing between the microsphere and the taper is controlled by electromechanical three-dimensional (3D) X-Y-Z translation stages with a $20 \mathrm{~nm}$ resolution. WGMs are excited by a wavelength-tunable laser (Agilent 81960A, wavelength near $1550 \mathrm{~nm}$ ) through the taper and detected by a photodiode detector.

Figure 2 shows the experimental setup of the proposed SLM, a narrow-bandwidth fiber ring laser stabilized by the taper-coupled DMC. The fiber ring cavity consists of a $980 \mathrm{~nm}$ pump laser diode (LD), a 980/1550 nm wavelengthdivision multiplexer (WDM), an isolator (ISO), an erbiumdoped fiber (EDF) with a length of $1.5 \mathrm{~m}$, a 90:10 fiber coupler, a polarization controller (PC) and the taper-coupled DMC. The pump LD is injected into the EDF through the $980 \mathrm{~nm}$ port of the WDM. The two microspheres are placed in parallel on opposite sides of the fiber taper, acting as a SLM narrowbandwidth mode-selector in the fiber ring cavity. The optical coupler is used to offer feedback and laser output for detection: with a $90 \%$ feedback into the ring cavity and a $10 \%$ output, measured by an optical spectrum analyzer (OSA), with a resolution of $0.02 \mathrm{~nm}$ (YOKOGAWA, AQ6370D).

\section{Theoretical AnALysis}

\section{A. Light Propagation Schematic in the Taper-Coupled DMC}

Figure 3 shows the schematic of the light propagation inside the taper-coupled DMC, in which, E1 is the incident light intensity, E2 is the WGM light intensity in microsphere 1 (above), E3 is the WGM light intensity in microsphere 2 (below), E4 is the output light intensity. Light is coupled into microsphere 1 via the taper, then coupled into microsphere 2 via the same fiber taper, and vice versa. The WGM resonant effect is enhanced inside the two microspheres through paralleled coupling via the same fiber taper and results in WGM spectra with a narrowed $3 \mathrm{~dB}$ bandwidth (BW). The theoretical analysis is given below.

\section{B. The Vernier Effect in the Taper-Coupled DMC}

The Vernier effect has been explored in various active microcavities [12]-[14]. For the SMC, its FSR is given as follows:

$$
F=\lambda^{2} c /\left(n_{e f f} \cdot L\right)
$$

where $n_{\text {eff }}$ is the effective refractive index, $\lambda_{c}$ is the resonant wavelength, $L$ is the cavity round-trip length. Different microsphere cavities have different FSR values due to their different diameters $\left(D^{\prime} s\right)$. The FSR of the DMC is given as [15]:

$$
F=N F_{1}=(N+1) F_{2}
$$

where $F_{1}$ and $F_{2}$ are the FSRs of the two cavities, $N$ is a positive integer, then we obtain:

$$
F=F_{1} F_{2} /\left|F_{2}-F_{1}\right|
$$

From (3) we see that due to the Vernier effect, the tapercoupled DMC has a $F_{2} /\left|F_{2}-F_{1}\right|$ times larger FSR than the SMC, which is better for side-mode suppression and narrow-bandwidth SLM selection as compared to the SMC.

\section{Comparison Between the SMC and the DMC}

The mode-field patterns in the microspheres are simulated by Finite-Difference Time-Domain (FDTD) method previously reported in [16]. Here we detail the simulation parameters of the taper-coupled DMC: The diameters of the two microspheres are the same: D1 $=\mathrm{D} 2=35 \mu \mathrm{m}$. The diameters chosen in the simulation are significantly smaller than those in the experiment. Thus, for phase-matched coupling, the taper diameter simulated is different $(1.4 \mu \mathrm{m})$ from that employed in the experiment, which is about $3 \sim 4 \mu \mathrm{m}$. The gap spacing between the microsphere and the taper is set to $0.1 \mu \mathrm{m}$ to achieve phase-matched coupling. The material refractive indices of the taper and the microsphere are 1.46. The light source is transverse-electric (TE) polarized. Fig. 4(a) shows the simulated transmission spectra of the SMC and the DMC. The simulation grid size is $52.3 \mathrm{~nm}$ and the resulting spectral resolution is $0.002 \mathrm{~nm}$. Figs. 4(b) and (c) show the simulated WGM intensity distributions of the SMC and the DMC. We can see from Fig. 4(a) that due to the Vernier effect in the DMC where the resonances of each microsphere coincide, the WGM oscillation is strengthened and results in a WGM spectrum with an increased $\mathrm{Q}$ factor and a narrowed $\mathrm{BW}$, which is narrowed from $0.495 \mathrm{~nm}$ to $0.351 \mathrm{~nm}$, compressed by about $29.3 \%$, as compared to that of the SMC. The ER of the DMC's WGM spectrum is about $45 \%$ higher than that of the SMC. Thus, the DMC has a better mode-selection performance than the SMC. Fig. 4(b) shows the WGM intensity distribution of the SMC at $1551.22 \mathrm{~nm}$. Fig. 4(c) shows the WGM intensity distribution of the DMC at $1550.96 \mathrm{~nm}$. 


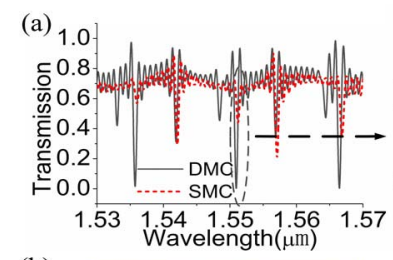

(b)

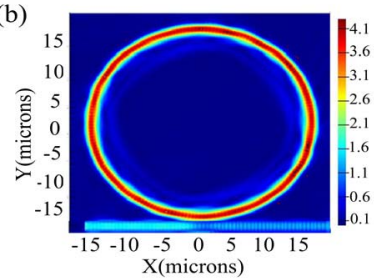

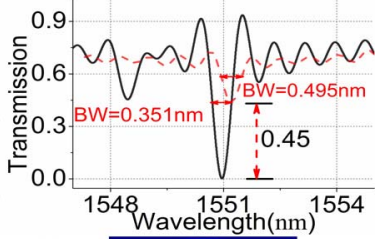

(c)

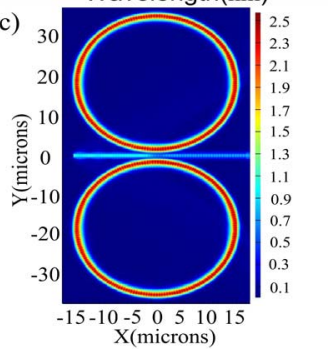

Fig. 4. (a) Comparison of the simulated WGM spectra of the DMC and the SMC. Inset: zoom-in-view WGM spectra. (b), (c) Simulated WGM intensity distributions of (b) the SMC at the wavelength of $1551.22 \mathrm{~nm}$ and of (C) the $\mathrm{DMC}$ at the wavelength of $1550.96 \mathrm{~nm}$.

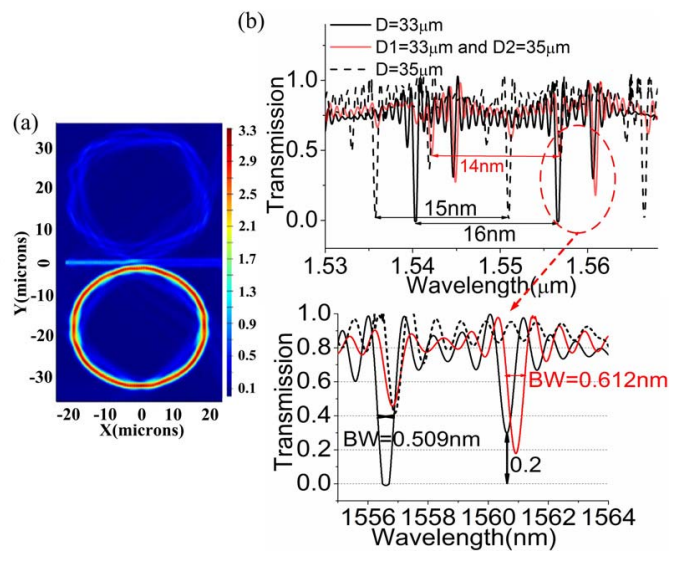

Fig. 5. (a) Simulated WGM intensity distribution of the DMC with different diameters at $1556.81 \mathrm{~nm}$. (b) Comparison of the simulated WGM spectra of the DMC with the same and different D's. Inset: zoom-in-view WGM spectra.

\section{Microsphere Size Dependence for the DMC Resonances}

Figure 5(a) shows the simulated WGM intensity distribution of the DMC for microspheres with different diameters (D1 $=33 \mu \mathrm{m}, \mathrm{D} 2=35 \mu \mathrm{m})$. The intensity color scale is in linear scale. Different resonant wavelengths between the two microspheres make it difficult to achieve phase matching between the DMC and the taper. In Fig. 5 (a), a WGM at $1556.81 \mathrm{~nm}$ is only excited in the microsphere located below the taper. The WGM field intensity is mainly distributed in a single microsphere. Fig. 5(b) shows the comparison of WGM spectra of the DMC, with the microspheres having the same diameters $(D=33 \mu \mathrm{m}, D=35 \mu \mathrm{m})$ and different diameters (D1 $=33 \mu \mathrm{m}, \mathrm{D} 2=35 \mu \mathrm{m})$, respectively. It can be seen clearly that when the two microspheres have the same $D$ of $33 \mu \mathrm{m}$, the WGM spectra have a $20 \%$ higher ER, a narrower BW and a larger FSR. Also, it can be seen from Fig. 5(b) that, for the cases that the microspheres are having the same diameters, the smaller $D=33 \mu \mathrm{m}$ case results in a larger FSR as compared to the larger $D=35 \mu \mathrm{m}$ case, which is in good accordance with Equation (1).

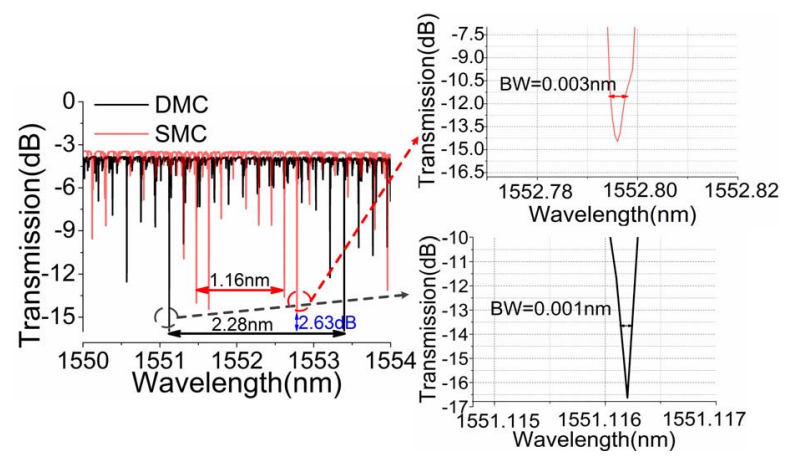

Fig. 6. Comparison of the measured WGM spectra of the SMC and the DMC. Insets: zoom-in-view WGM transmission dips.

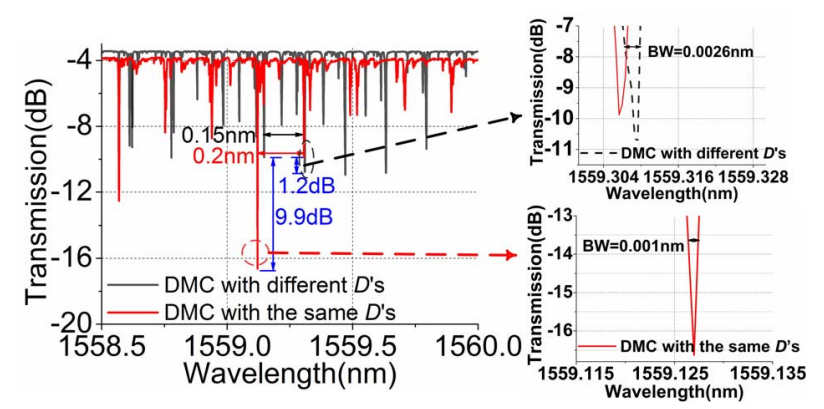

Fig. 7. Measured WGM spectra of the DMC with different and the same diameters. Insets: zoom-in-view WGM transmission dips of the DMC with different D's (above) and the same D's (below).

\section{EXPERIMENTAL RESULTS AND DisCUSSIONS}

In order to produce stabilized WGM spectra for stable fiber laser operation, over-coupling is applied in our experiments, the microsphere cavities are in touch with the fiber taper with a diameter of about $3 \sim 4 \mu \mathrm{m}$, which is phase-matched well with the microspheres. Figure 6 shows the comparison between the experimentally measured WGM spectra of the SMC and the DMC. The spectral resolution of the transmission measurements is $0.5 \mathrm{pm}$. The equatorial diameter of the SMC is $\sim 198.4 \mu \mathrm{m}$, and the equatorial diameters of the double microspheres are $\sim 183.4 \mu \mathrm{m}$ and $198.3 \mu \mathrm{m}$, respectively (due to fabrication imperfections, D1 and D2 are different by nearly $10 \%$, which is comparable to the fractional difference between $\mathrm{D} 1=33 \mu \mathrm{m}$ and $\mathrm{D} 2=35 \mu \mathrm{m}$ in simulations. This may allow the experimental results and the simulation results of the DMC to be qualitatively compared). It can be seen clearly that the DMC can produce a TE-polarized WGM spectrum with a $(2.63 \mathrm{~dB})$ higher ER, a narrower $\mathrm{BW}$ and a $(1.12 \mathrm{~nm})$ larger FSR as compared to those of the SMC, which is also in good accordance with our theoretical analysis.

Figure 7 shows the comparison of the measured WGM spectra within a wavelength span between the DMC with almost the same diameters (198.4 $\mu \mathrm{m}, 198.3 \mu \mathrm{m})$ and different diameters $(183.4 \mu \mathrm{m}$ and $198.3 \mu \mathrm{m})$. The DMC with the same $D^{\prime} s$ produces a TE-polarized WGM spectrum with a wider FSR and a higher ER (about $8.7 \mathrm{~dB}$ ) as compared to the DMC with different $D$ 's (about $1.2 \mathrm{~dB}$ ) near the wavelength of $1559.31 \mathrm{~nm})$. There are significantly more high-order modes near $1559.1 \mathrm{~nm}$ for the DMC with microspheres of 


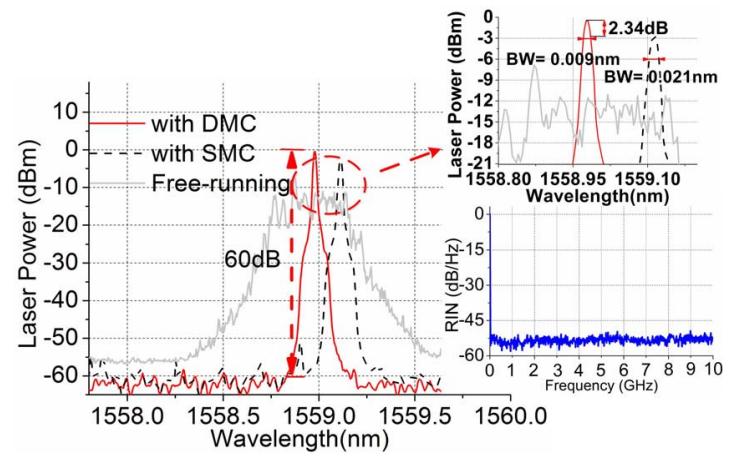

Fig. 8. Comparison of the measured laser spectra with the DMC, the SMC, and the free-running laser operation. Insets: zoom-in-view spectra (above) and measured radio-frequency (RF) spectrum (below) of the proposed singlelongitudinal-mode fiber ring laser using self-homodyne method.
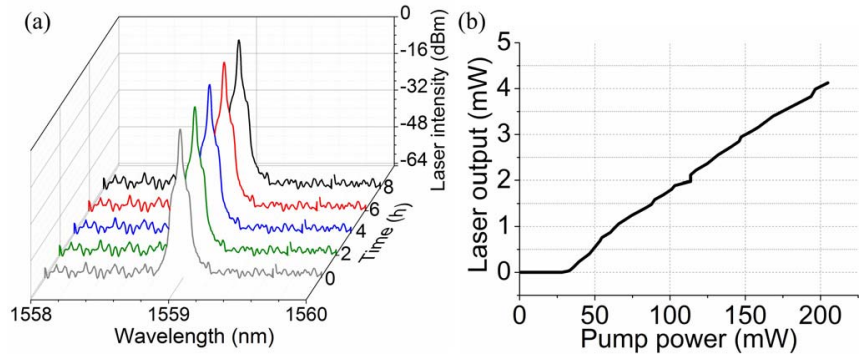

Fig. 9. Measured laser output of: (a) Mode-hopping-free SLM laser output measured for 8 hours with a pump power of $100 \mathrm{~mW}$; (b) Measured laser output versus pump power.

different diameters. Thus, the DMC with the same microsphere diameters has a better mode -selection performance, which is in good accordance with the simulation results.

Figure 8 shows the measured laser spectra based on: the DMC, the SMC and the free-running fiber laser with a pump power of about $100 \mathrm{~mW}$. Using the DMC (with the same parameters as in Fig. (6)) as the mode-selector, we observe the narrowest 3dB-BW of less than $0.01 \mathrm{~nm}$ for the SLM laser spectrum, with a highest signal-to-background ratio of about $60 \mathrm{~dB}$. The SLM operation is verified by using a frequency spectrum analyzer (Rohde \& Schwarz, FSV30) without beating frequency appeared, as shown in the inset (below). The fiber laser system is placed on a floating vibration-isolated platform and the coupling position is kept stable through a specially designed package. As compared to the SMC method, the DMC method can effectively improve the laser signal-tobackground ratio (by $2.34 \mathrm{~dB}$ ), compress the laser linewidth (by $0.012 \mathrm{~nm}$ ), and improve the stability (by $8 \mathrm{hr}$ ) of the laser output.

Fig. 9 (a) shows the stable SLM laser output measured for 8 hours of using DMC as the mode-selector. When the laser was pumped at about $100 \mathrm{~mW}$, no mode hopping was observed when the laser outputs are monitored and compared at selected times. The wavelength and output power of the laser is relatively stable. The laser threshold is about $32.2 \mathrm{~mW}$, having a slope efficiency of about 2.4\%, as shown in Fig. 9(b).

\section{CONCLUSiON}

We proposed and demonstrated a SLM, narrow-bandwidth fiber laser using the taper-coupled DMC as the mode-selector inside a fiber ring cavity. As compared with the SMC, the DMC produces WGM spectra with a higher ER, a higher SMSR, a narrower BW as well as a larger FSR, which achieves a SLM and a highly stabilized laser output with a narrower BW of $<0.01 \mathrm{~nm}$, a higher signal-to-background ratio of about $60 \mathrm{~dB}$, and a higher laser efficiency as compared to using the SMC for mode selection. This fiber laser could find potential applications in optical communications and sensing systems.

\section{REFERENCES}

[1] L. Yu et al., "Widely tunable narrow-linewidth lasers using self-injection DBR lasers," IEEE Photon. Technol. Lett., vol. 27, no. 1, pp. 50-53, Jan. 1, 2015.

[2] H. Al-Taiy, N. Wenzel, S. Preußler, J. Klinger, and T. Schneider, "Ultra-narrow linewidth, stable and tunable laser source for optical communication systems and spectroscopy," Opt. Lett., vol. 39, no. 20, pp. 5826-5829, Oct. 2014.

[3] E. Luvsandamdin et al., "Micro-integrated extended cavity diode lasers for precision potassium spectroscopy in space," Opt. Exp., vol. 22, no. 7, pp. 7790-7798, Apr. 2014

[4] C.-H. Yeh, H.-Z. Chen, J.-Y. Chen, and C.-W. Chow, "Use of fiber Bragg grating (FBG) for stable and tunable erbium-doped fiber ring laser with single-longitudinal-mode (SLM) output," Laser Phys., vol. 25, no. 11, p. 115101, Nov. 2015.

[5] H. Wan et al., "An injection-locked single-longitudinal-mode fiber ring laser with cylindrical vector beam emission," IEEE Photon. J., vol. 9, no. 1, Feb. 2017, Art. no. 7100608.

[6] J. Chen, Y. Zhao, Y. Zhu, S. Liu, and Y. Ju, "Narrow line-width ytterbium-doped fiber ring laser based on saturated absorber," IEEE Photon. Technol. Lett., vol. 29, no. 5, pp. 439-441, Mar. 1, 2017.

[7] H. Wan, W. Jiang, Y. Gong, C. Pan, and X. Sun, "Single-longitudinalmode fiber ring laser stabilized by tandem all-fiber Fabry-Pérot microcavities," IEEE Photon. Technol. Lett., vol. 24, no. 5, pp. 404-406, Mar. 1, 2012.

[8] H. Wan, Z. Wu, Z. Lu, X. Sun, and J. Wang, "A watts-level singlelongitudinal-mode kHz-linewidth fiber laser based on a gain-controlled active compound cavity," Laser Phys., vol. 24, no. 10, p. 105114, Oct. 2014.

[9] M. C. Collodo, F. Sedlmeir, B. Sprenger, S. Svitlov, L. J. Wang, and H. G. L. Schwefel, "Sub-kHz lasing of a $\mathrm{CaF}_{2}$ whispering gallery mode resonator stabilized fiber ring laser," Opt. Exp., vol. 22, no. 16, pp. 19277-19283, Aug. 2014.

[10] A. Chiasera et al., "Spherical whispering-gallery-mode microresonators," Laser Photon. Rev., vol. 4, no. 3, pp. 457-482, Jul. 2009.

[11] H. Wan, H. Li, H. Zhu, and J. Xu, "Tunable, ultra-narrow-band optical filter based on a whispering gallery mode hybrid-microsphere," Chin. Opt. Lett., vol. 14, no. 11, pp. 112302-112305, Nov. 2016.

[12] L. Shang, L. Xu, and L. Liu, "Single-frequency coupled asymmetric microcavity laser," Opt. Lett., vol. 33, no. 10, pp. 1150-1152. 2008.

[13] T. Grossmann et al., "Polymeric photonic molecule super-mode lasers on silicon," Light Sci. Appl., vol. 2, no. 5, p. e82, 2013.

[14] V. D. Ta, R. Chen, and H. Sun, "Coupled polymer microfiber lasers for single mode operation and enhanced refractive index sensing," Adv. Opt. Mater, vol. 2, no. 3, pp. 220-225, 2014.

[15] D. Dai, "Highly sensitive digital optical sensor based on cascaded high-Q ring-resonators," Opt. Exp., vol. 17, no. 26, pp. 23817-23822, Nov. 2009.

[16] J.-D. Lin et al., "Optical bistability in GaInAsP/InP coupled-circular resonator microlasers," Opt. Lett., vol. 36, no. 17, pp. 3515-3517, 2011. 